\title{
EDITORIAL
}

\section{European report card issued}

\author{
The publication of the Annual Epidemiological Report from the ECDC gives a snapshot \\ of the most important communicable disease threats faced by the EU.
}

December 2013 saw the publication of the Annual Epidemiological Report from the European Centre for Disease Prevention and Control (ECDC) ${ }^{1}$. The report details the surveillance and epidemiological intelligence data for 2011 and 2012, respectively, that were gathered from 30 countries (the 28 European Union (EU) member states as well as Iceland and Norway), and presents a comprehensive picture of the state of European health with respect to the 52 communicable diseases that fall under the watchful eye of the ECDC.

Healthcare-associated infections (HCAIs) feature prominently as the report incorporates the results of the agency's first EU-wide point prevalence survey (PPS) to estimate the overall burden of HCAIs and hospital-based antimicrobial consumption (which were also published separately earlier in 2013 (REF. 2)). Information gathered from 947 European acute-care hospitals according to a standardized methodology and covering $\sim 230,000$ patients was used in the final report. The PPS revealed that, every day, an estimated one in 18 patients (of $\sim 80,000$ patients in total) has a HCAI and that one in three patients is receiving at least one antimicrobial drug.

The report also presents data on antimicrobial resistance (AMR) that were collected by the European Antimicrobial Resistance Surveillance Network. These data support the ongoing worrying trends in the AMR burden that, rightly, received a great deal of media and political attention in 2013. The incidence of Escherichia coli that is resistant to third-generation cephalosporins significantly increased between 2008 and 2011 in 18 of 28 countries, with most isolates testing positive for extended-spectrum $\beta$-lactamases (ESBLs); moreover, the incidence of multidrug-resistant $E$. coli (that is, organisms that are resistant to fluoroquinolones and aminoglycosides, in addition to third-generation cephalosporins) increased in 13 of 28 countries during the same period.

The incidence of Klebsiella pneumoniae that is resistant to third-generation cephalosporins also increased between 2008 and 2011 in ten of 25 countries, with up to $100 \%$ of isolates reported to be ESBL-positive. Multidrug resistance in K. pneumoniae remains a key concern, and the data showed that multidrug resistance was widespread in K. pneumoniae that had been isolated from patients in southern, central and eastern
European countries. Given that this increase in multidrug resistance puts increasing pressure on carbapenems as the 'antibiotics of last resort', it is worrying to see that the percentage of $K$. pneumoniae that exhibit carbapenem resistance is also increasing across the continent as this severely limits further treatment options for these infections.

However, there was some positive news - although still an important problem, the prevalence of methicillinresistant Staphylococcus aureus has remained stable, or is decreasing, in most European countries. Interestingly, for many resistant infections there was a distinct northsouth differential, with lower levels of AMR reported in northern European countries. This probably reflects differences in antimicrobial usage or infection-control regimens between northern and southern Europe and should be explored further.

The report is not all about AMR, of course, and the chapter on vector-borne diseases makes fascinating reading. Many reported vector-borne diseases, such as malaria, are imported into the EU from endemic countries. However, the report highlights the increasing risk of a shift to indigenous transmission in countries where the appropriate vectors are present, as has been observed for West Nile virus, which is now endemic in the southeast of Europe. For example, in 2012, Greece reported that 16 of 76 malaria cases were the result of local rather than imported transmission. Elsewhere, October 2012 signalled the start of an ongoing outbreak of dengue fever in Madeira. More than 2,000 cases have been reported so far, in what is the first serious indigenous outbreak of this disease in the EU. Clearly, vector-borne infectious diseases will be a growing public health risk in Europe in the future.

This annual report card for European health makes interesting reading. Overall, although progress has been made in some areas, there is definite room for improvement; our verdict: a B-.

\footnotetext{
1. European Centre for Disease Prevention and Control. Annual Epidemiological Report [online], http://www.ecdc.europa.eu/en/ publications/Publications/annual-epidemiological-report-2013.pdf publications/Publications/annual-epidemiological-report-2013.
(2013).
European Centre for Disease Prevention and Control. Point
Prevalence Survey of Healthcare-associated Infections and Antimicrobial Use in European Acute Care Hospitals [online], http:// www.ecdc.europa.eu/en/publications/Publications/healthcareassociated-infections-antimicrobial-use-PPS.pdf (2013).
} 